\title{
Men's experiences of coping with their wives' breast cancer involved focusing on the cancer and treatment and focusing on family to keep life going
}

Hilton BA, Crawford JA, Tarko MA. Men's perspectives on individual and family coping with their wives'breast cancer and
chemotherapy. West J Nurs Res 2000 Jun;22:438-59.

QUESTION: What were the experiences of men as individuals, partners, and fathers when their wives had chemotherapy for breast cancer?

Design

Qualitative naturalistic inquiry.

\section{Setting}

Vancouver, British Columbia, Canada.

\section{Participants}

10 English speaking, white male partners (age range $39-58$ y) of women who had chemotherapy for breast cancer in the previous 2 years and had $\geqslant 1$ child living at home were identified through purposive sampling.

\section{Methods}

Men participated in individual, semi-structured interviews of 1-2 hours, which were conducted by male nurses. Interviews focused on men's perceptions of their experience and their family's experience of coping with breast cancer. Interviews were audiotaped and transcribed, and analysed using the constant comparative method.

\section{Main findings}

Men's coping was characterised by 2 major themes: focusing on their wives' illness and care and focusing on the family to keep life going. 9 subthemes were identified, each of which predominantly reflected one of the 2 major themes, although most reflected both themes to some extent. Focusing on a wife's illness and care was reflected in men's efforts to understand, assist with decision making, get through treatment, and deal with the healthcare system. 3 subthemes were associated with this major theme. Being there meant that men wanted to be physically present and emotionally supportive. They wanted to accompany their wives to appointments, access information and services, and provide care. Most felt unprepared and did not know what to do or how to behave. Men relied on healthcare professionals to give competent care, and to provide support and assistance. They wanted to be treated with respect and compassion and to have their views considered and respected. Being informed and contributing to decision making involved learning about cancer, assisting with and supporting their wives' decisions, and informing children, family, and friends.

The second major theme was focusing on family to keep life going amid the stress and disruption from the illness. 6 subthemes related to this theme. Most men were challenged by trying to keep patterns normal, as they struggled to keep family routines and activities intact, especially for the children. For family life to continue, men had to help out and rely on others to undertake household and child care activities. Men felt stretched and overwhelmed, particularly when helping at home was unfamiliar. Men spoke about trying to be positive and to have positive people around them. They were strength- ened by their wives' positive attitudes, although spouses often protected each other by not sharing fears and emotions. Most men helped by putting [their] self on hold. Initially, they were totally consumed by the cancer, its treatment, and keeping family life going; many automatically put their own needs as secondary to their wives' needs. Men talked about adapting work life to balance work and home demands. Some adapted work patterns and wished they had taken more time off, whereas others saw work as an escape. Managing finances was an added stress as men tried to deal with unexpected reduced incomes and increased expenses.

\section{Conclusion}

Men's experiences of coping with their partners' breast cancer and treatment were reflected in the 2 major themes of focusing on their wives' illness and care and focusing on family to keep life going.
Source of funding: Canadian Breast Cancer Foundation.

For correspondence: Professor B A Hiltom University of British Columbia, School of Nursing T201-2211 Wesbrook Mall, Vancouver, British Columbia V6T 2B5, Canada.Fax +1604 8227466.

\section{COMMENTARY}

The study by Hilton et al contributes to our understanding of men's experiences of coping with their wives' breast cancer. Two other qualitative studies have found similar results. ${ }^{12}$ Zahlis and Shands found that male partners of women with breast cancer negotiated the illness experience by becoming educated about the cancer, adapted their lifestyle to balance home and work responsibilities, were sensitive to their wives' emotional and physical needs, and tried to minimise illness effects. ${ }^{1}$ Wilson and Morse identified a buffering process used by husbands to protect their wives (eg, filtering and eliminating daily stressors and monitoring responses to chemotherapy). ${ }^{2}$

Although the sample in the study by Hilton et al comprised husbands of women who had received chemotherapy for breast cancer, the findings also have implications for the family. The findings clearly highlight the importance of seeing the family as a unit throughout the cancer illness, in terms of coping, role change, distress, and concern. It also highlights the complexities of partners dealing with illness and treatment while trying to maintain a sense of family normality.

Many nurses who work in cancer care will see familiarities with issues raised in their own practice. This study offers direction for the development and subsequent evaluation of nursing interventions to assist men in similar situations. Potential interventions could focus on providing support individually and in peer groups, ensuring adequate provision of information, facilitating men's involvement in the care and treatment process, encouraging individual and family sessions on communication and techniques for dealing with feelings, and providing counselling to address men's needs and concerns related to issues such as supporting their wives and children, household management, asking for help, and accessing resources and services. Furthermore, exploratory research is also needed to extend our understanding of the experiences of partners and other family members coping with different types of cancer diagnoses and treatments. Susie Pearce RGN, MSc Clinical Nurse Specialist Practice Development and Research, Cancer Care UCL Hospitals NHS Trust London, UK

1 Zahlis EH, Shands ME. Breast cancer: demands of the illness on the patient's partner. Journal of Psychosocial Oncology 1991;9:75-93.

2 Wilson S, Morse JM. Living with a wife undergoing chemotherapy. Image J Nurs Sch 1991;23:78-84. 